\title{
Clarithromycin increases linezolid exposure in multidrug-resistant tuberculosis patients
}

\author{
Mathieu S. Bolhuis ${ }^{1}$, Richard van Altena ${ }^{2}$, Dick van Soolingen ${ }^{3,4,5}$, \\ Wiel C.M. de Lange², Donald R.A. Uges' ${ }^{1}$, Tjip S. van der Werf ${ }^{6}$, \\ Jos G.W. Kosterink ${ }^{1,7}$ and Jan-Willem C. Alffenaar ${ }^{1}$
}

\begin{abstract}
Affiliations: 'Dept of Hospital and Clinical Pharmacy, University of Groningen, University Medical Centre Groningen, Groningen, ${ }^{2}$ Tuberculosis Centre Beatrixoord, University of Groningen, University Medical Centre Groningen, Haren, ${ }^{3}$ Dept of Pulmonary Medicine, Radboud University Nijmegen Medical Centre, Nijmegen, ${ }^{4}$ Department of Medical Microbiology, Radboud University Nijmegen Medical Centre, Nijmegen, ${ }^{5}$ Unit Mycobacteria, Diagnostic Laboratory for Bacteriology and Parasitology, National Institute for Public Health and the Environment (RIVM), Bilthoven, ${ }^{6}$ Dept of Internal Medicine and Pulmonary Disease and Tuberculosis, University of Groningen, University Medical Centre Groningen, Groningen, and ${ }^{7}$ Dept of Pharmacy, Pharmacotherapy and Pharmaceutical Care, University of Groningen, Groningen, The Netherlands.
\end{abstract}

Correspondence: M.S. Bolhuis, University Medical Centre Groningen, Dept of Hospital and Clinical Pharmacy, University of Groningen, PO Box 30.001, 9700 RB Groningen, The Netherlands. E-mail: m.s.bolhuisđumcg.nl

ABSTRACT The use of linezolid for the treatment of multidrug-resistant tuberculosis is limited by doseand time-dependent toxicity. Recently, we reported a case of pharmacokinetic drug-drug interaction between linezolid and clarithromycin that resulted in increased linezolid exposure. The aim of this prospective pharmacokinetic study is to quantify the effect of clarithromycin on the exposure of linezolid.

Subjects were included in an open-label, single-centre, single-arm, fixed-order pharmacokinetic interaction study. All subjects received $300 \mathrm{mg}$ linezolid twice daily during the entire study, consecutively co-administered with $250 \mathrm{mg}$ and $500 \mathrm{mg}$ clarithromycin once daily. Steady-state serum curves of linezolid and clarithromycin were analysed using validated methods, and differences between pharmacokinetic parameters were calculated.

Linezolid exposure increased by a median (interquartile range) of $44 \%(23-102 \%, p=0.043)$ after coadministration of $500 \mathrm{mg}$ clarithromycin $(n=5)$ compared to baseline, whereas $250 \mathrm{mg}$ clarithromycin had no statistically significant effect. Co-administration was well tolerated by most patients; none experienced severe adverse effects. One patient reported common toxicity criteria grade 2 gastrointestinal adverse events.

In this study, we showed that clarithromycin significantly increased linezolid serum exposure after combining clarithromycin with linezolid in multidrug-resistant tuberculosis patients. The drug-drug interaction is possibly P-glycoprotein-mediated. Due to large interpatient variability, therapeutic drug monitoring is advisable to determine individual effect size.

@ERSpublications

Clarithromycin significantly increased linezolid serum exposure in multidrug-resistant tuberculosis patients http://ow.ly/oYGK1

For editorial comments see page 1449 .

Received: Jan 042013 | Accepted after revision: March 042013 | First published online: March 212013

Clinical trial: This study is registered at clinicaltrials.gov with identifier number NCT01521364.

Support statement: This study was financially supported by Stichting Beatrixoord Noord-Nederland (Groningen, The Netherlands).

Conflict of interest: Disclosures can be found alongside the online version of this article at www.erj.ersjournals.com

Copyright @ERS 2013 


\section{Introduction}

Multidrug-resistant tuberculosis (MDR-TB) is an infectious disease of major concern, especially in countries with a high TB burden [1-3]. Treatment of MDR-TB poses challenges such as designing an effective second-line antituberculosis regimen, entailing a combination of multiple drugs and a long duration of treatment [4]. This translates into an intensive phase of $\geqslant 8$ months and total treatment duration of $\geqslant 20$ months as recommended by the World Health Organization (WHO) [5]. In the intensive phase, treatment of MDR-TB should consist of at least four second-line antituberculosis drugs likely to be effective. Additional drugs from group 5, such as linezolid and clarithromycin, may be used, but their efficacy in the treatment of MDR-TB is unclear [5]. Unfortunately, knowledge on the efficacy in the treatment of MDR-TB with these drugs is scarce.

Linezolid is a promising antimicrobial agent for the treatment of MDR-TB. However, evidence on the treatment of MDR-TB with linezolid is limited. Efficacy against Mycobacterium tuberculosis has been shown in vitro [6], in animals [7] and in patients [8-10]. A recent meta-analysis confirms this efficacy, but shows the necessity of caution in the prescription of linezolid due to toxicity; almost $60 \%$ of all analysed patients experienced adverse events [11]. Adverse events, such anaemia (38\%), peripheral neuropathy (47\%), gastrointestinal side-effects/symptoms (17\%), optic neuritis (13\%) and thrombocytopenia (12\%), have all been reported and limit the use of linezolid [11]. Reducing the dose of linezolid has been evaluated in an attempt to reduce toxicity [12]. A dose of $\leqslant 600 \mathrm{mg}$ linezolid daily resulted in lower frequency of adverse events than a dose of $>600 \mathrm{mg}$ daily ( $47 \%$ versus $75 \%$ ), thereby enabling longer treatment duration [11].

Clarithromycin has a less prominent place in the treatment of MDR-TB. The minimal inhibitory concentration (MIC) of M. tuberculosis was thought to be well in excess of achievable serum concentrations based on 12 strains of M. tuberculosis [13]. However, lower MICs have been observed $\left(<2 \mathrm{mg} \cdot \mathrm{L}^{-1}\right)$ and clarithromycin shows concentrations in epithelial lining fluid that are often higher than in serum, enabling clarithromycin to be added to treatment regimens [14]. Several group 5 drugs, e.g. linezolid and clarithromycin, may need to be combined in a single MDR-TB treatment regimen, although little is known on the drug-drug interactions of these agents. Drug-drug interactions could compromise the efficacy of treatment regimens or could increase toxicity through reduced or increased exposure, respectively.

Recently, we reported a pharmacokinetic drug-drug interaction between linezolid and clarithromycin that resulted in increased linezolid exposure [15]. Increased serum linezolid concentrations could lead to toxicity, such as time- and dose-dependent severe myelosuppression and polyneuropathy. In a metaanalysis comparing a cohort treated with $>600 \mathrm{mg}$ linezolid per day with a cohort treated with $\leqslant 600 \mathrm{mg}$ per day, there was a higher probability of anaemia (60\% versus $23 \%)$, leukopenia (17\% versus $2 \%$ ) and gastrointestinal symptoms $(29 \%$ versus $8 \%)$ in the cohort that received $>600 \mathrm{mg}$ linezolid [11]. Such toxicity could lead to the need to cease treatment with linezolid, severely limiting the treatment options left. Therefore, the aim of this prospective pharmacokinetic study was to quantify the effect of clarithromycin on the exposure of linezolid in adult MDR-TB patients hospitalised at the Tuberculosis Centre Beatrixoord (Haren, the Netherlands).

\section{Method \\ Study design}

This study was an open-label, prospective single-centre, single-arm, fixed-order, interventional pharmacokinetic interaction study. The study was performed at the Tuberculosis Centre Beatrixoord. All study subjects received standard care for their MDR-TB and comorbidities. Treatment of MDR-TB was based on the WHO guidelines [5] individualised for each patient included.

The primary objective was to quantify linezolid area under the concentration-time curve from 0 to $12 \mathrm{~h}$ (AUC0-12) without clarithromycin and with $250 \mathrm{mg}$ and $500 \mathrm{mg}$ clarithromycin once daily. Secondary objectives were to compare pharmacokinetic parameters of linezolid and clarithromycin between different dosing combinations and to describe the tolerability and safety of co-administration of clarithromycin and linezolid in MDR-TB patients.

All patients gave written informed consent. The study protocol was approved by the medical ethical review committee of the University Medical Centre Groningen (Groningen, the Netherlands). The study was registered at clinicaltrials.gov (identifier number NCT01521364).

\section{Subjects}

All study subjects were aged $\geqslant 18$ years and were diagnosed with MDR-TB, confirmed using standard microbiological culture methods. The criteria for exclusion were based on the contraindications and known drug-drug interactions as mentioned in the summary of product characteristics of linezolid and clarithromycin $[16,17]$. Subjects were excluded from the study if they were pregnant or lactating; had 
previously shown hypersensitivity to linezolid, any macrolide antibiotics or any of the excipients of linezolid or clarithromycin; had hypokalaemia; or concomitantly received P-glycoprotein modulators. Drug sensitivity testing (DST) was performed at the Dutch National Mycobacterial Reference Laboratory (National Institute for Public Health and the Environment (RIVM), Bilthoven, The Netherlands) using the Middlebrook 7H10 agar dilution method.

\section{Treatment}

All patients received linezolid $300 \mathrm{mg}$ every $12 \mathrm{~h}$. In previous studies, we showed that this dose resulted in seemingly effective serum concentrations with a median (interquartile range (IQR)) AUC0-12 of $57.6 \mathrm{mg} \cdot \mathrm{h} \cdot \mathrm{L}^{-1}\left(38.5-64.2 \mathrm{mg} \cdot \mathrm{h} \cdot \mathrm{L}^{-1}\right)$ and $\mathrm{AUC} 0-12 / \mathrm{MIC}$ ratios of 452 (343-513) [12]. Clarithromycin was added to therapy at a dosage of $250 \mathrm{mg}$ and $500 \mathrm{mg}$ once daily consecutively during 2 weeks in a fixed order (fig. 1). From three cases at the Tuberculosis Centre Beatrixoord, of which one case is published [15], it was expected that $500 \mathrm{mg}$ clarithromycin would result in an approximately doubled linezolid exposure, matching the exposure of linezolid at the labelled dose of $600 \mathrm{mg}$ twice daily.

Full linezolid pharmacokinetic curves were recorded at baseline (after 1 week of linezolid without clarithromycin), after receiving linezolid with $250 \mathrm{mg}$ clarithromycin for 2 weeks and after linezolid with $500 \mathrm{mg}$ clarithromycin for 2 weeks (fig. 1). A trough sample was obtained after a washout period of 1 week, during which the patients only received linezolid besides their standard treatment, but no clarithromycin.

Sample size was derived from AUCs in a previous study in MDR-TB patients [12] and from the relative large increase of exposure observed in three cases ([15]; two cases unpublished). To reach a desired power of $80 \%$, a sample size of at least five patients was calculated using $\mathrm{G}^{\star}$ Power 3.1 (Heinrich Heine Universität, Düsseldorf, Germany). A dropout rate of $15 \%$ was estimated based on previous studies at the Tuberculosis Centre Beatrixoord. To compensate for this estimated dropout, seven patients were included.

\section{Experimental procedures}

The baseline linezolid pharmacokinetic curve and the trough after a 1-week washout period were obtained at steady state, which is reached after $\sim 3$ days [16]. Pharmacokinetic curves after co-administration of linezolid and clarithromycin were assessed at steady state after 2 weeks, allowing the pharmacokinetic interaction to develop fully [18]. Blood samples were collected before and 1, 2, 3, 4, 8 and $12 \mathrm{~h}$ after intake of medication. The second dose of linezolid was given directly after this last blood sample. The patients did not receive standardised meals, but were allowed to eat a regular breakfast, reflecting common clinical practice, since food does not influence linezolid exposure [19]. Adherence was ensured through a directly observed inpatient treatment programme.

\section{Serum concentrations}

Blood samples were drawn and after centrifuging serum samples were stored at $-20^{\circ} \mathrm{C}$ until analysis. Linezolid and clarithromycin serum concentrations were analysed using validated high-performance liquid chromatography tandem mass-spectrometry methods [20, 21].

\section{Tolerability and safety}

The patients were clinically observed by nurses and attending physicians. Routine checks including blood tests were carried out at least weekly as part of continued standard care including monitoring for hyperlactataemia and haematological abnormalities such as thrombocytopaenia and anaemia. All patients received epoetine- $\alpha$ (Eprex; Janssen-Cilag, Leiden, The Netherlands) pre-emptively in a dose of 2000 IU twice a week to prevent anaemia as part of standard care. Gastrointestinal side-effects were determined using the common toxicity criteria (CTC) and were scored grades $0-4$ [22]. Routine testing of neurotoxic adverse events through electromyogram or vibration sense monitoring were not carried out during the 6-week study, since these effects have been reported to occur after a median (range) of 16 weeks (10-111 weeks) [23]. In case of clinical suspicion of peripheral neuropathy, a neurologist was consulted, as is common practice at the Tuberculosis Centre Beatrixoord. Furthermore, patients receiving linezolid were examined by an ophthalmologist once monthly, which is also common practice in this centre.

\section{Pharmacokinetic and statistical analysis}

The main study parameter linezolid AUC0-12, and secondary study parameters clearance, elimination constant and elimination half-life are calculated using trapezoidal rule in the Kinfit software (MWPharm 3.60; Mediware, Groningen, the Netherlands) [24]. The pharmacokinetic parameters of linezolid and clarithromycin are described. The maximal serum concentration $(\mathrm{Cmax})$ was defined as the highest observed serum concentration and $\mathrm{Cmin}$ was defined as the concentration before intake of medication. 


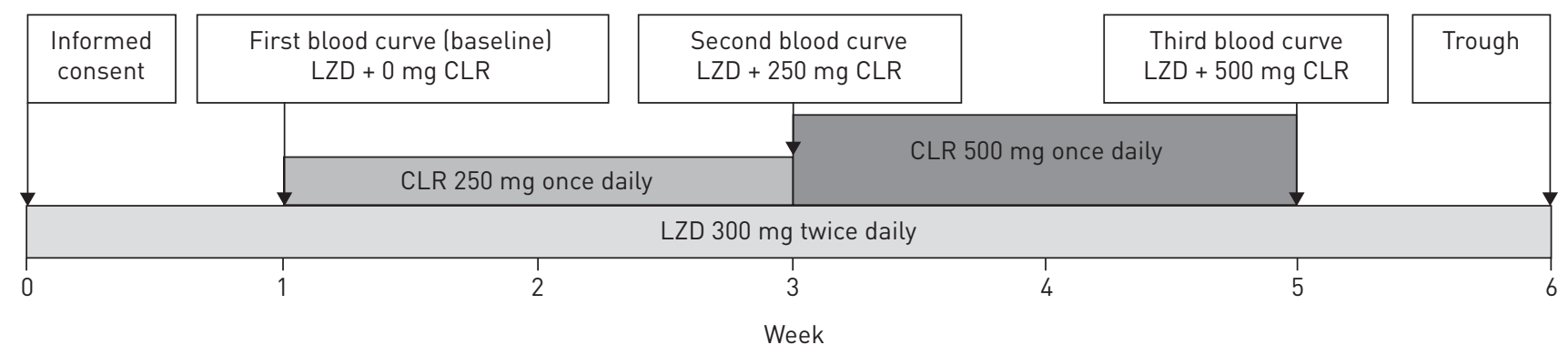

FIGURE 1 Study design, showing dosing and sampling schedules of linezolid (LZD) and clarithromycin (CLR). Before, during and after the study patients received standard medical care and treatment for multidrug-resistant tuberculosis.

The hypothesis that the median of differences of AUC0-12 of linezolid at baseline compared to AUC0-12 after co-administration with either $250 \mathrm{mg}$ or $500 \mathrm{mg}$ clarithromycin is equal to zero was tested using the related-samples Wilcoxon signed rank test. Secondary pharmacokinetic parameters from the three curves were compared using the same related-samples Wilcoxon signed rank test. The nonparametric ANOVA Friedman test was used to test the dose dependency of the effect of clarithromycin on linezolid exposure. All statistical evaluations were performed using SPSS 20 (SPSS, Chicago, IL, USA).

\section{Results}

\section{Patient characteristics}

From December 2011 to October 2012, 16 patients with possible MDR-TB were admitted to the Tuberculosis Centre Beatrixoord. Two of these 16 patients were aged $<18$ years, one patient was pregnant, one patient was participating in another study, and one patient's planned period of admission was too short. This left 11 patients available for formal screening. Four patients were not included in the study for

\section{TABLE 1 Baseline demographics and results from drug susceptibility testing}

\begin{tabular}{|c|c|}
\hline Parameter & Value \\
\hline Subjects & 5 \\
\hline Age years & $35.0(23-65)$ \\
\hline Male & $4(80)$ \\
\hline Weight kg & $66.8(55.2-78.5)$ \\
\hline Height m & $1.74(1.67-1.82)$ \\
\hline Body mass index $\mathrm{kg} \cdot \mathrm{m}^{-2}$ & $22.1(17.1-26.2)$ \\
\hline \multicolumn{2}{|l|}{ Ethnicity } \\
\hline African & 3 \\
\hline Caucasian & 1 \\
\hline Asian & 1 \\
\hline HIV-positive & 1 \\
\hline \multicolumn{2}{|c|}{$\begin{array}{l}\text { Isolate resistant to drug based on drug } \\
\text { susceptibility testing }\end{array}$} \\
\hline Ethambutol & $3 / 5$ \\
\hline Isoniazid & $5 / 5$ \\
\hline Pyrazinamide ${ }^{\#}$ & $2 / 4$ \\
\hline Rifampicin & $5 / 5$ \\
\hline Streptomycin & $4 / 5$ \\
\hline Capreomycin & $1 / 5$ \\
\hline Amikacin & $0 / 5$ \\
\hline Ciprofloxacin & $1 / 5$ \\
\hline Clarithromycin ${ }^{\#}$ & $2 / 3$ \\
\hline Clofazimine & $0 / 3$ \\
\hline Linezolid & $0 / 5$ \\
\hline Moxifloxacin & $1 / 5$ \\
\hline Protionamide $\#$ & $1 / 4$ \\
\hline Rifabutin & $4 / 5$ \\
\hline
\end{tabular}

Data are presented as $\mathrm{n}$, mean (range), $\mathrm{n}(\%)$ or $\mathrm{n} / \mathrm{N}$. DST: drug susceptibility testing. ${ }^{\#}$ : not available for all isolates of the included patients. 


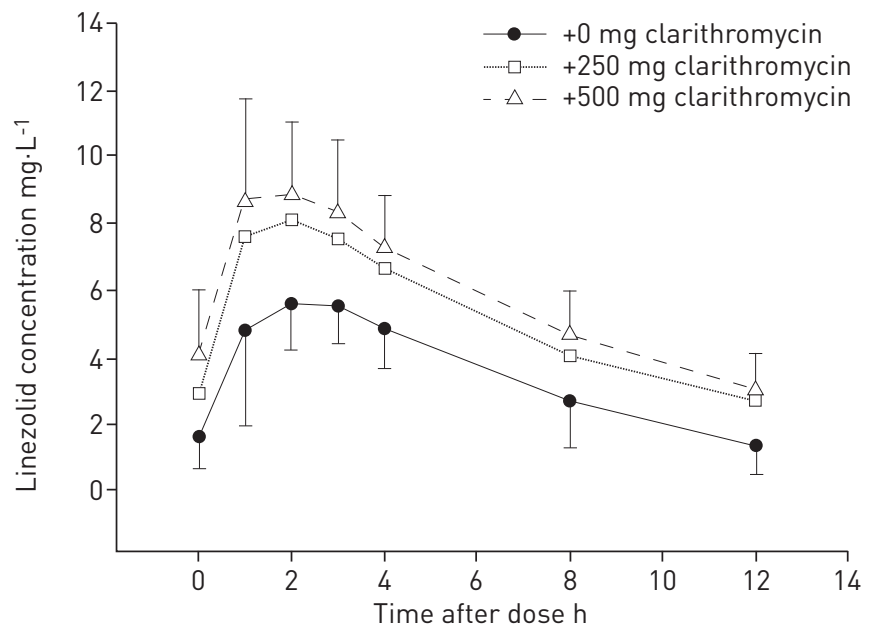

FIGURE 2 Mean linezolid concentration-time curves in serum $(n=5)$ without clarithromycin, with $250 \mathrm{mg}$ clarithromycin and with $500 \mathrm{mg}$ clarithromycin. Error bars: SD. For visual purposes, error bars for linezolid with $250 \mathrm{mg}$ clarithromycin have been omitted.

various reasons. In one patient DST revealed normal sensitivity. In another patient venous blood samples were not obtained due to venous access problems; rendering the collection of three full pharmacokinetic curves impossible. A third patient was included in another study and the last patient was deemed too psychologically unstable to comply with the study protocol. Seven hospitalised patients were included in the study, five of whom were suitable for evaluation. One of the included patients dropped out of the study in the fourth week due to medical reasons. The patient had a fever and was nauseous, probably due to an infected venous access port and possibly combined with side-effects of clarithromycin and other anti-TB medication, such as moxifloxacin. Another patient could not be evaluated due to a logistical problem with the study medication. The two patients who dropped out of the study were excluded from all analyses.

Patient baseline demographics and results from the drug susceptibility testing are presented in table 1 . The mean (range) age of included subjects was 35 years (23-65 years) and the mean (range) weight was $66.8 \mathrm{~kg}$ $(55.2-78.5 \mathrm{~kg})$. One of the patients was HIV-positive and was treated with emtricitabin/tenofovir and raltegravir. Three patients originated from Somalia, one from Turkey and one from the Netherlands.

\section{Pharmacokinetic and statistical analysis}

From all patients suitable for evaluation $(n=5)$, three full pharmacokinetic curves in serum were available. The mean serumw concentration-time curves are shown in figure 2. The baseline median (IQR) AUC0-12 of linezolid of $36.3 \mathrm{mg} \cdot \mathrm{h} \cdot \mathrm{L}^{-1}\left(33.2-46.3 \mathrm{mg} \cdot \mathrm{h} \cdot \mathrm{L}^{-1}\right)$ in patients with a body weight of median $71.5 \mathrm{~kg}$ (IQR $56.8-72.0 \mathrm{~kg}$ ), is lower than the median (IQR) AUC0-12 of linezolid of $57.6 \mathrm{mg} \cdot \mathrm{h} \cdot \mathrm{L}^{-1}\left(38.5-64.2 \mathrm{mg} \cdot \mathrm{h} \cdot \mathrm{L}^{-1}\right)$ from a previous study with patients with a body weight median (IQR) of $58.3 \mathrm{~kg}$ (52.7-62.8 $\mathrm{kg}$ ) [12]. Linezolid concentrations in serum increased after co-administration of clarithromycin compared to baseline, but display a large standard deviation. There appears to be no effect on time Cmax is reached, i.e. the maximal time $\left(t_{\max }\right)$.

Pharmacokinetic parameters of linezolid and clarithromycin are presented in table 2. Compared to baseline, the median AUC0-12 of linezolid increased statistically significantly after co-administration with $500 \mathrm{mg}$ clarithromycin $(\mathrm{p}=0.043)$, but not after the co-administration of $250 \mathrm{mg}$ clarithromycin $(\mathrm{p}=0.686)$. After the co-administration of linezolid with $500 \mathrm{mg}$ clarithromycin, the median (IQR) AUC0-12 of linezolid increased by $44 \%$ (23-102\%) compared to baseline. Furthermore, the administration of $500 \mathrm{mg}$ clarithromycin statistically significantly increased the Cmax of linezolid by a median (IQR) of $48 \%$ (35-103\%, $\mathrm{p}=0.043)$; however, no significant increase was observed with the Cmin of linezolid, which had a median (IQR) of $50 \%(44-189 \%, \mathrm{p}=0.080)$ when compared to the baseline. There was no statistically significant difference in linezolid half-life after co-administration of $500 \mathrm{mg}$ clarithromycin with linezolid compared to linezolid alone $(p=0.138)$. Linezolid clearance and elimination constant decreased statistically nonsignificantly when linezolid and $500 \mathrm{mg}$ clarithromycin were co-administered compared to baseline (both $\mathrm{p}=0.08$ ). No dose-dependent effect of clarithromycin on the linezolid exposure could be detected using the Friedman test $(\mathrm{p}=0.091)$.

\section{Tolerability and safety}

Co-administration of linezolid and clarithromycin was well tolerated by most patients. None of the patients experienced severe adverse events, such as anaemia, peripheral neuropathy, optic neuritis or 
TABLE 2 Pharmacokinetic parameters of linezolid and clarithromycin

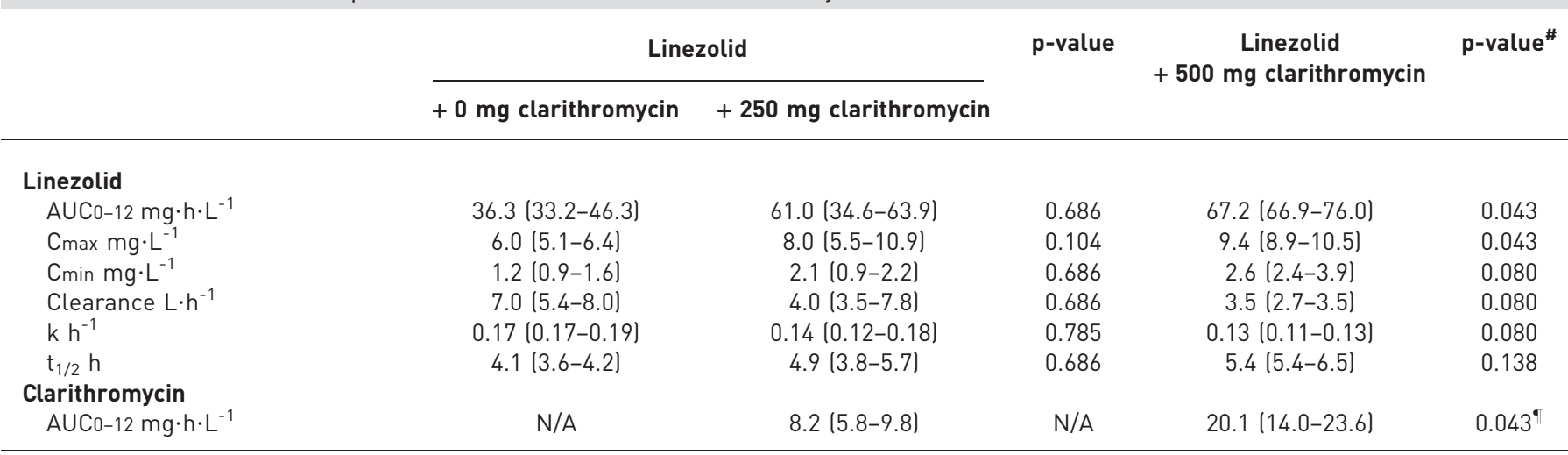

Data are presented as median (interquartile range). $n=5$. AUC0-12: area under the concentration-time curve from 0 to $12 \mathrm{~h} ; \mathrm{C}_{\text {max: }}$ maximal serum concentration; $\mathrm{Cmin}$ : the concentration before intake of medication; $\mathrm{k}$ : elimination constant. N/A: not applicable. \#: p-values comparing parameters from co-administration of linezolid with $500 \mathrm{mg}$ clarithromycin to baseline; ${ }^{\top}$ : p-value comparing AUC0-12 of $500 \mathrm{mg}$ with $250 \mathrm{mg}$ clarithromycin.

thrombocytopenia. One patient experienced CTC grade 2 gastrointestinal side-effects 3 days after the start of administration of $500 \mathrm{mg}$ clarithromycin once daily.

\section{Discussion}

In this study, we showed that clarithromycin significantly increased linezolid serum AUC0-12 after combining clarithromycin with linezolid in MDR-TB patients. After 2 weeks of co-administration of linezolid with clarithromycin $500 \mathrm{mg}$ once daily, the Cmax of linezolid increased significantly by $\sim 50 \%$. Combining linezolid with clarithromycin in a dose of $500 \mathrm{mg}$ once daily resulted in a significantly higher AUC0-12 of linezolid with a median of $44 \%$. None of the patients experienced any severe adverse events. However, it should be noted that patients pre-emptively received epoetine- $\alpha$ as part of standard care, potentially obscuring anaemia as a side-effect.

Besides our recent report on the interaction between clarithromycin and linezolid, there are no other reports on this pharmacokinetic drug interaction. In fact, one of the few known drug interactions of linezolid to date is with rifampicin. Rifampicin, a well-known inducer of P-glycoprotein and cytochrome P450 enzymes, decreases linezolid serum levels in critically ill patients [25]. Another study confirmed this finding in healthy volunteers [26, 27]. GEBHART et al. [25] suggest the interaction to be mediated by Pglycoprotein, since an in vitro study has shown that linezolid is not metabolised by cytochrome P450 enzymes [28]. The interaction of linezolid and clarithromycin could also be mediated by P-glycoprotein, since clarithromycin is a well-known cytochrome P450 3A (CYP3A)4 inhibitor and a potent inhibitor of Pglycoproteins [29]. P-glycoprotein is a membrane efflux transporter enzyme that is highly expressed in a variety of tissues including the intestine, liver and kidney [30]. Inhibition of the P-glycoprotein efflux pump by clarithromycin could result in the increased levels of linezolid, possibly a P-glycoprotein substrate, through inhibition of P-glycoprotein at the intestinal site as well as the renal site. P-glycoprotein polymorphism could explain some of the interpatient variation that we observed. However, in a recent study Gandelman et al. [27] refer to unpublished data on file from Pfizer suggesting that linezolid is not a P-glycoprotein substrate. Their hypothesis for the observed interaction between linezolid and rifampicin is that a large increase in expression of CYP3A, which typically has a small contribution $(0.7-10.5 \%)$ to linezolid clearance, could cause a small decrease in linezolid exposure [27]. Further research on the exact mechanism of the drug-drug interaction is needed.

Co-administration of clarithromycin and linezolid resulted in a near statistically significant decrease of clearance and elimination constant of linezolid compared to baseline. This might suggest the inhibition of CYP3A or renal or hepatic P-glycoprotein efflux transporter pumps. However, decreased clearance might not solely explain the observed increase of linezolid exposure. Unfortunately, due to the limited number of samples during the absorption phase, it is impossible to adequately compare data on absorption constant and tmax. Since patients did not receive intravenous linezolid, data on bioavailability is not available. It is therefore difficult to draw conclusions on involvement of inhibition of intestinal P-glycoprotein efflux transporters, which could result in increased absorption. 
The increase of linezolid exposure after co-administration with clarithromycin has possible implications for clinical practice. The higher linezolid AUC0-12 could result in toxicity of linezolid, an agent that often causes adverse events, such as time- and dose-dependent severe myelosuppression and polyneuropathy. Severe adverse events often necessitate the cessation of effective anti-MDR-TB treatment, leaving few alternatives. Dose reduction of linezolid could prevent toxicity. However, care should be taken to assure adequate linezolid exposure and added information on whether linezolid exposure is too high, too low or in the therapeutic range, could prove helpful. Therapeutic drug monitoring could help in assessing the linezolid dose after dose reduction [12], especially since the observed drug-drug interaction shows a large interpatient variability. In limited-resource settings, dried blood spot sampling could resolve logistical problems encountered with conventional therapeutic drug monitoring [31].

After evaluation of the combination of clarithromycin and linezolid in a larger population and over a longer period of time, clarithromycin could eventually even be used as a booster for linezolid, comparable to the use of low-dose ritonavir as a booster to improve protease inhibitor exposure in combined antiretroviral therapy. The relatively cheap clarithromycin could reduce the dose of the expensive linezolid while the same exposure is maintained, thereby leaving the risk of toxicity unaltered. Since the highest prevalence of MDR-TB is found in countries with limited resources, such a booster strategy could make treatment with linezolid feasible for a larger group of patients. Such a cost reduction could even contribute to the call for making global MDR-TB control affordable [32]. Further research on WHO group 5 drugs such as linezolid, besides evaluation of new drugs such as delaminid [33] or old drugs such as co-trimoxazole [34], is of great importance.

In conclusion, we showed a $44 \%$ increase of linezolid AUC0-12 after co-administration of linezolid with clarithromycin in a dose of $500 \mathrm{mg}$ daily in MDR-TB patients. The pharmacokinetic interaction between linezolid and clarithromycin is suggested to be mediated by P-glycoprotein. Further research in a larger cohort is needed to provide insight into the observed interpatient variation, perhaps caused by Pglycoprotein polymorphism. Until effect size is predictable, possibly with help of P-glycoprotein polymorphism testing, therapeutic drug monitoring is advisable to determine individual effect size. The drug-drug interaction we showed in this study is an important step towards making the effective anti-TB drug linezolid available through cost reduction in less affluent settings where MDR-TB is highly prevalent.

\section{References}

1 Migliori GB, Dara M, de Colombani P, et al. Multidrug-resistant tuberculosis in Eastern Europe: still on the increase? Eur Respir J 2012; 39: 1290-1291.

2 Jenkins HE, Plesca V, Ciobanu A, et al. Assessing spatial heterogeneity of MDR-TB in a high burden country. Eur Respir J 2013; 42: 1291-1301.

3 Skrahina A, Hurevich $\mathrm{H}$, Zalutskaya A, et al. Alarming levels of drug-resistant tuberculosis in Belarus: results of a survey in Minsk. Eur Respir J 2012; 39: 1425-1431.

4 Falzon D, Gandhi N, Migliori GB, et al. Resistance to fluoroquinolones and second-line injectable drugs: impact on MDR-TB outcomes. Eur Respir J 2013; 42: 156-168.

5 Guidelines for the Programmatic Management of Drug-Resistant Tuberculosis. 2011 Update. Geneva, World Health Organization, 2011.

6 Prammananan T, Chaiprasert A, Leechawengwongs M. In vitro activity of linezolid against multidrug-resistant tuberculosis (MDR-TB) and extensively drug-resistant (XDR)-TB isolates. Int J Antimicrob Agents 2009; 33: 190-191. Cynamon MH, Klemens SP, Sharpe CA, et al. Activities of several novel oxazolidinones against Mycobacterium tuberculosis in a murine model. Antimicrob Agents Chemother 1999; 43: 1189-1191.

8 De Lorenzo S, Alffenaar JW, Sotgiu G, et al. Efficacy and safety of meropenem-clavunate added to linezolidcontaining regimens in the treatment of MDR-/XDR-TB. Eur Respir J 2013; 41: 1386-1392.

9 Lee M, Lee J, Carroll MW, et al. Linezolid for treatment of chronic extensively drug-resistant tuberculosis. N Engl J Med 2012; 367: 1508-1518.

10 Migliori GB, Eker B, Richardson MD, et al. A retrospective TBNET assessment of linezolid safety, tolerability and efficacy in multidrug-resistant tuberculosis. Eur Respir J 2009; 34: 387-393.

11 Sotgiu G, Centis R, D’Ambrosio L, et al. Efficacy, safety and tolerability of linezolid containing regimens in treating MDR-TB and XDR-TB: systematic review and meta-analysis. Eur Respir J 2012; 40: 1430-1442.

12 Alffenaar JW, van Altena R, Harmelink IM, et al. Comparison of the pharmacokinetics of two dosage regimens of linezolid in multidrug-resistant and extensively drug-resistant tuberculosis patients. Clin Pharmacokinet 2010; 49: 559-565.

13 Truffot-Pernot C, Lounis N, Grosset JH, et al. Clarithromycin is inactive against Mycobacterium tuberculosis. Antimicrob Agents Chemother 1995; 39: 2827-2828.

14 Honeybourne D, Kees F, Andrews JM, et al. The levels of clarithromycin and its 14-hydroxy metabolite in the lung. Eur Respir J 1994; 7: 1275-1280.

15 Bolhuis MS, van Altena R, Uges DR, et al. Clarithromycin significantly increases linezolid serum concentrations. Antimicrob Agents Chemother 2010; 54: 5418-5419.

16 Pfizer. Zyvoxid. Product Information. College ter Beoordeling van Geneesmiddelen/Medicines Evaluation Board. http://db.cbg-meb.nl/IB-teksten/h26569.pdf Date last accessed: June 22, 2010. Date last updated: October 19, 2011.

17 Sandoz. Clarithromycin. Product Information. College ter Beoordeling van Geneesmiddelen/Medicines Evaluation Board, 2004. http://db.cbg-meb.nl/IB-teksten/h30789.pdf Date last accessed: June 22, 2010. Date last updated: May $23,2011$. 
Stockley IH. General considerations and an outline survey of some basic interaction mechanisms. In: Stockley IH, ed. Stockley's Drug Interactions. 6th Edn. London, Pharmaceutical Press, 2002; pp. 1-14.

19 Islinger F, Dehghanyar P, Sauermann R, et al. The effect of food on plasma and tissue concentrations of linezolid after multiple doses. Int J Antimicrob Agents 2006; 27: 108-112.

20 Harmelink IM, Alffenaar JW, Wessels AM, et al. A rapid and simple liquid chromatography-tandem mass spectrometry method for the determination of linezolid in human serum. Eur J Hosp Pharm 2008; 14 : 5-8.

21 de Velde F, Alffenaar JW, Wessels AM, et al. Simultaneous determination of clarithromycin, rifampicin and their main metabolites in human plasma by liquid chromatography-tandem mass spectrometry. J Chromatogr B Analyt Technol Biomed Life Sci 2009; 877: 1771-1777.

22 Trotti A, Colevas AD, Setser A, et al. CTCAE v3.0: development of a comprehensive grading system for the adverse effects of cancer treatment. Semin Radiat Oncol 2003; 13: 176-181.

23 Anger HA, Dworkin F, Sharma S, et al. Linezolid use for treatment of multidrug-resistant and extensively drugresistant tuberculosis, New York City, 2000-06. J Antimicrob Chemother 2010; 65: 775-783.

24 Proost JH, Meijer DK. MW/Pharm, an integrated software package for drug dosage regimen calculation and therapeutic drug monitoring. Comput Biol Med 1992; 22: 155-163.

25 Gebhart BC, Barker BC, Markewitz BA. Decreased serum linezolid levels in a critically ill patient receiving concomitant linezolid and rifampin. Pharmacotherapy 2007; 27: 476-479.

26 Egle H, Trittler R, Kümmerer K, et al. Linezolid and rifampin: drug interaction contrary to expectations? Clin Pharmacol Ther 2005; 77: 451-453.

27 Gandelman K, Zhu T, Fahmi OA, et al. Unexpected effect of rifampin on the pharmacokinetics of linezolid: in silico and in vitro approaches to explain its mechanism. J Clin Pharmacol 2011; 51: 229-236.

28 Wynalda MA, Hauer MJ, Wienkers LC. Oxidation of the novel oxazolidinone antibiotic linezolid in human liver microsomes. Drug Metab Dispos 2000; 28: 1014-1017.

29 Eberl S, Renner B, Neubert A, et al. Role of p-glycoprotein inhibition for drug interactions: evidence from in vitro and pharmacoepidemiological studies. Clin Pharmacokinet 2007; 46: 1039-1049.

30 Thiebaut F, Tsuruo T, Hamada H, et al. Cellular localization of the multidrug-resistance gene product Pglycoprotein in normal human tissues. Proc Natl Acad Sci USA 1987; 84: 7735-7738.

$31 \mathrm{Vu}$ DH, Bolhuis MS, Koster RA, et al. Dried blood spot analysis for therapeutic drug monitoring of linezolid in patients with multidrug-resistant tuberculosis. Antimicrob Agents Chemother 2012; 56: 5758-5763.

32 Loddenkemper R, Sotgiu G, Mitnick CD. Cost of tuberculosis in the era of multidrug resistance: will it become unaffordable? Eur Respir J 2012; 40: 9-11.

33 Skripconoka V, Danilovits M, Pehme L, et al. Delamanid improves outcomes and reduces mortality in multidrugresistant tuberculosis. Eur Respir J 2013; 41: 1393-1400.

34 Alsaad N, van Altena R, Pranger AD, et al. Evaluation of co-trimoxazole in treatment of multidrug-resistant tuberculosis. Eur Respir J 2013; 42: 504-512. 